\title{
Synaptic Vesicle Movements Monitored by Fluorescence Recovery after Photobleaching in Nerve Terminals Stained with FM1-43
}

\author{
A. W. Henkel, ${ }^{1}$ L. L. Simpson, ${ }^{2}$ R. M. A. P. Ridge, ${ }^{3}$ and W. J. Betz ${ }^{1}$ \\ ${ }^{1}$ Department of Physiology, University of Colorado Medical School, Denver, Colorado 80262, 2Departments or Medicine \\ and Pharmacology, Jefferson Medical College, Philadelphia, Pennsylvania 19107-6799, and '3epartment of Physiology, \\ Bristol University School of Medical Sciences, Bristol BS8 1TD, United Kingdom
}

We used the fluorescence recovery after photobleaching technique to monitor movements of synaptic vesicles in top views of living frog motor nerve terminals that had been prestained with the fluorescent dye FM1-43. In each experiment, a small portion of a single stained vesicle cluster was bleached with a laser and monitored subsequently for signs of recovery as neighboring, unbleached vesicles moved into the bleached region. In resting terminals, little or no recovery from photobleaching occurred. Repetitive nerve stimulation, which caused all fluorescent spots to grow dim as dye was released from exocytosing vesicles, did not promote recovery from photobleaching. Pretreatment with botulinum toxin (type A, C, or D) blocked exocytosis and destaining, but intense nerve stimulation still did not cause significant recovery in bleached regions. These results suggest that lateral movements of synaptic ves-

In nerve terminals, synaptic vesicles exist in compact clusters near sites of exocytosis. During repetitive nerve activity, vesicles move to the presynaptic membrane and undergo exocytosis. The mechanisms governing vesicle clustering and mobilization are only partly understood. It is not known, for example, whether vesicles in resting terminals mix freely, as granules in chromaffin cells seem to do (Terakawa et al., 1993), or are immobile, as suggested by studies of synapsin I (for review, see DeCamilli et al,, 1990). Nor is it known how synaptic vesicles, once mobilized by nerve activity, move to the presynaptic membrane. In the present work, we have studied these and other questions by measuring the intracellular movement of the fiuorescent dye FM1-43. This dye stains membranes of recycled synaptic vesicles in motor nerve terminals and is released during exocytosis (Henkel et al., 1996). For example, if a frog muscle is exposed to FM1-43 during nerve stimulation, synaptic vesicles that undergo exocytosis take up the dye during endocytosis, and clusters of recycled, stained vesicles appear as bright fluorescent spots 1-3 $\mu \mathrm{m}$ in diameter (each comprising hundreds of stained vesicles) distributed at regular

Received Feb. 5, 1996; revised March 8, 1996; accepted March 12, 1996

This work was supported by National Institutes of Health Research Grants NS23466 to W.J.B. and R.M.A.P.R., NS10207 to W.J.B., and NS22153 to L.L.S.; an MDA research grant to W.J.B.; USDOA contracts DAMD1795C-0048 and -5004 to L.L.S.; and a Human Frontier Science Fellowship to A.W.H. Steve Fadul provided unfailing assistance in all phases of these experiments. We thank Dr. M. Sheetz (Durham) for his encouragement in the early stages of this work, and Dr. W. Almers (Heidelberg) for insightful comments during the course of the work and for helpful suggestions on the manuscript.

Correspondence should be addressed to W.J. Betz, Department of Physiology, University of Colorado Medical School, Denver, CO 80262.

Copyright $\mathcal{C} 1996$ Socicty for Neuroscience $0270-6474 / 96 / 163960-08 \$ 05.00 / 0$ icles are restricted severely in both resting and stimulated nerve terminals.

We tested for laser-induced photodamage in several ways. Bleached regions could be restained fully with $\mathrm{FM} 1-43$, and these restained regions could be destained fully by nerve stimulation. Partially bleached regions could be destained, although the rate of destaining was lower than normal. Brisk recovery from photobleaching occurred after treatment with okadaic acid, which disrupts synaptic vesicle clusters and causes vesicles to spread throughout the nerve terminal. These results suggest that vesicle translocation and recycling machinery was intact in photobleached regions.

Key words: photobleach recovery; FPR; FRAP; synaptic vesicles; okadaic acid; FM1-43; exocytosis intervals along the length of the terminals (Betz and Bewick, 1992, 1993; Betz et al., 1992a; Betz et al., 1993; Bewick and Betz, 1994; Henkel and Betz, 1995; Ribchester and Betz, 1994).

In the present study, we used the fluorescence recovery after photobleaching (FRAP) technique (Axelrod et al., 1976; Wade et al., 1986; Jacobson et al., 1991) to study synaptic vesicle movements within single vesicle clusters. We used a laser to photobleach a small segment of a fluorescent spot, and then we monitored the bleached region for signs of recovery as dye moved from neighboring (unbleached) territories into the bleached region. The results suggest that vesicles do not move freely either in resting or stimulated terminals.

\section{MATERIALS AND METHODS}

Experiments were performed on acutely dissected frog (Rana pipiens) cutaneus pectoris muscles. To stain nerve terminals, preparations were exposed to 2-4 $\mu$ M FM1-43 (Molecular Probes, Eugene, OR) dissolved in normal frog Ringer's while the nerve was stimulated electrically at $10 \mathrm{~Hz}$ for 4.5-5 min (Betz et al., 1992a). For imaging and photobleaching, we used a Biorad 600 laser scanning confocal microscope with a mixed-gas $\mathrm{Kr}$-Ar laser, fitted on an upright Nikon microscope with a Zeiss $40 \times$ water immersion objective lens. The following settings were used: $488 \mathrm{~nm}$ excitation line; $1 \%$ transmittance filter, photomultiplier tube gain manual and maximal, black level manual, zoom 3-5, scan speed normal or slow. The aperture usually was fully open. For processing and analysis, images were sent to a Silicon Graphics computer running software by G. W. Hannaway. For any series of images of the same terminal, all images were acquired and processed identically. For spot photobleaching, the "park" command was used to position the laser beam, and the shutter then was opened for $0.2-1.0 \mathrm{sec}$. For line photobleaching, 1000-3000 linescans were given to produce bleaching. Results from line photobleaching experiments were not significantly different from spot photobleaching ex- 

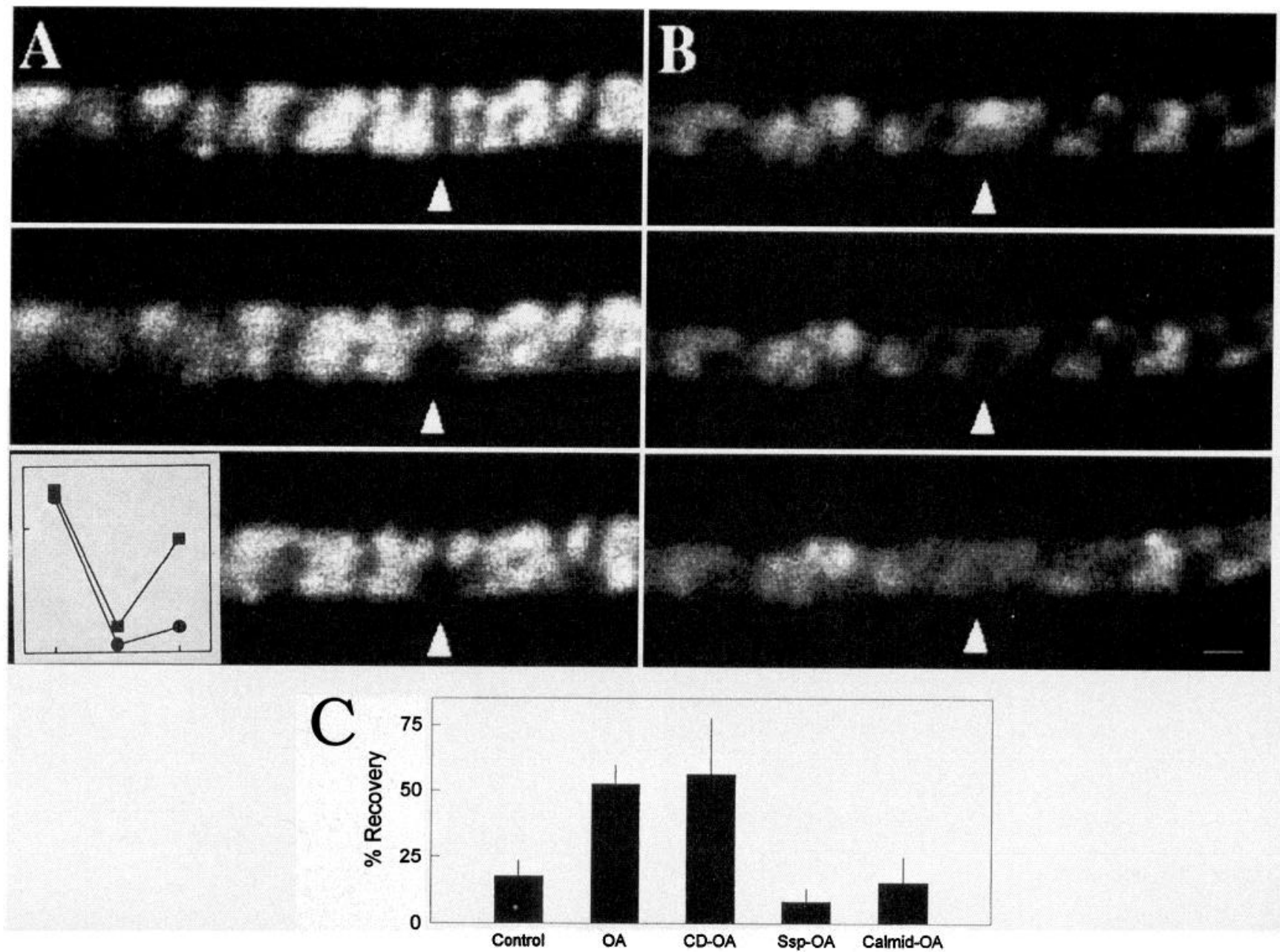

Figure 1. Okadaic acid promoted recovery from photobleaching in resting nerve terminals. $A$, Control nerve terminal before bleaching (top), immediately after bleaching (middle; the bleached region is marked with an arrowhead), and $28 \mathrm{~min}$ after bleaching (bottom). The bleached regions in the middle and bottom are almost identical, indicating that little or no dye moved from the neighboring, unbleached regions into the bleached region. Inset, The brightness $(y$-axis, not normalized) of the bleached spot is plotted for each of the three panels. Circles are from controls $(A)$; squares are from okadaic acid-treated terminals $(B)$. Data represent average brightness of three concentric circles (radii $0.2,0.4$, and $1.0 \mu \mathrm{m}$ ) centered on the bleached regions. Note that each region began at the same brightness and was bleached by a similar amount. Only the okadaic acid-treated preparation showed significant recovery. $B$, Nerve terminal treated with okadaic acid. These images are like those in $A$, except that okadaic acid (5 $\mu \mathrm{M}$ ) was added 40 min before bleaching (and was present throughout the experiment), and the last image was acquired only $5.4 \mathrm{~min}$ after bleaching. It is clear that significant recovery from photobleaching occurred relatively rapidly. Scale bar, $2 \mu \mathrm{m}$. $C$, Quantification of photobleach recovery. Bars show mean percent recovery $(+1$ SEM) from photobleaching for various treatments. In each case, measurements were made $25-30 \mathrm{~min}$ after photobleaching. The control and okadaic acid $(O A)$ results are like those illustrated in Figures 1 and 2. The third column $(C D-O A)$ shows that $\mathrm{CD}(20 \mu \mathrm{M}$ for $20 \mathrm{~min})$ did not block the recovery from photobleaching induced by okadaic acid. The last two columns (Ssp-OA and Calmid-OA) show that Ssp ( $2 \mu \mathrm{M}$ for $60 \mathrm{~min})$ and calmidozolium (Calmid) (10 $\mu \mathrm{M}$ for $30 \mathrm{~min})$ each blocked the action of okadaic acid.

periments and are not illustrated here. Only terminals on surface muscle fibers were studied.

Botulinum toxin serotype A was isolated and tested for potency as described previously (Simpson et al., 1988). Serotypes C and D were purchased from WAKO Fine Chemicals (Dallas, TX); their potency was confirmed with tests on both mouse (Simpson and Dasgupta, 1983) and frog (J. Coffield and L.L. Simpson, unpublished observations) nervemuscle preparations. FM1-43-loaded preparations were exposed to 2-10 $\mathrm{nM}$ botulinum toxin for $3-5 \mathrm{hr}$ at room temperature $\left(\sim 23^{\circ} \mathrm{C}\right)$; complete paralysis required exposure for $\sim 3 \mathrm{hr}$. The block of destaining was not reversible by prolonged washing.

The use of black widow spider venom was described elsewhere (Henkel and Betz, 1995). Briefly, the venom glands from Latrodectus mactans were purchased from Sigma (St. Louis, MO) and a crude extract prepared the day of the experiments. Solutions contained $\sim 0.3$ gland/ml Ringer's and were applied for 40-60 min.

Image orientation. We acquired only top views of nerve terminals. That is, the terminals that we imaged lay on the upper surface of muscle fibers.
Thus, the laser bleached columns of vesicles oriented primarily perpendicular to the presynaptic membrane. Consequently, all results concern only lateral movements of vesicles and dye. We attempted to perform experiments on terminals viewed from the side, but the dye spots were too thin (seldom more than $1 \mu \mathrm{m}$ ) to permit partial bleaching of a fluorescent spot viewed in this orientation, even in linescan mode.

Quantification of photobleach recovery. In some experiments, the overall brightness of images decreased slightly during the experiment. This image "fade" may have resulted from photobleaching during repeated image acquisition or from slight focus changes, or both. Usually, we made no compensation for such changes, although in some cases (Fig. 1) the brightness of a control region (outside the region of intended photobleaching) was measured and normalized to the first image in the series by adding a constant value to all pixels within the confines of the terminal.

We measured the brightness of the center of the bleached region before and at various times after photobleaching and calculated the percent of recovery. For example, if the average pixel value (brightness) of the bleached region was 200 before bleaching, 100 immediately after 

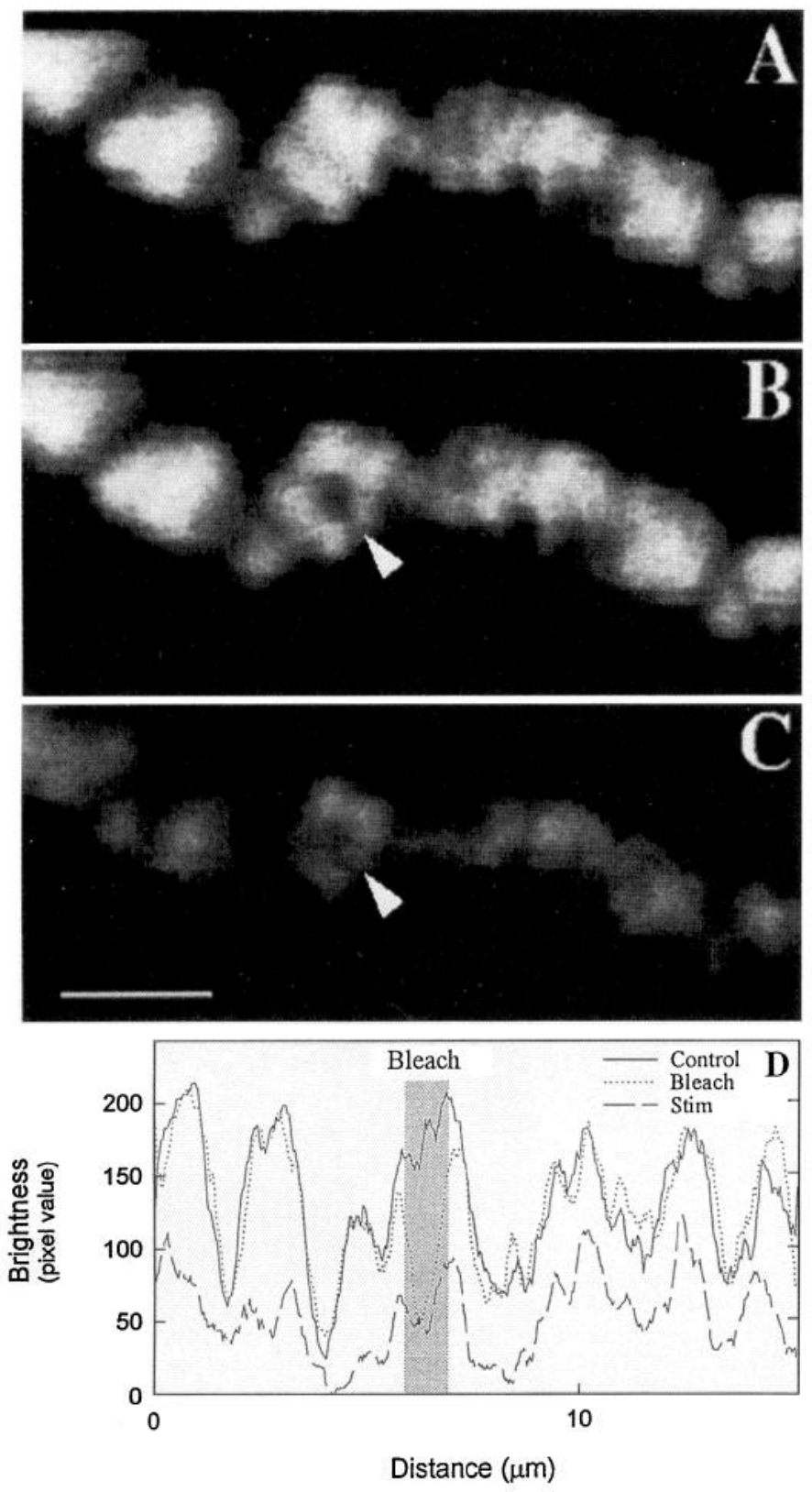

Figure 2. Nerve stimulation did not promote recovery from photobleaching. $A$, Image of nerve terminal immediately before photobleaching. $B$, Same terminal immediately after bleaching. Note the bleached center in the spot marked with an arrowhead. $C$, Same terminal after repetitive nerve stimulation $(10 \mathrm{~Hz}$ for $5 \mathrm{~min}$ ). All of the spots destained as a result of the nerve stimulation. The bleached spot is less evident as a result of the surrounding destaining. Scale bar, $4 \mu \mathrm{m}$. D, Brightness of pixels along a line drawn through the center of several adjacent spots for each image in $A-C(A$, solid line; $B$, dotted line; $C$, dashed line $)$. The bleached region is shaded. Note that nerve stimulation did not cause the bleached region to grow brighter, indicating that no net movement of dye into the bleached region occurred during the stimulation period.

bleaching, and 150 later, the recovery was $50 \%$. In addition, we measured the brightness of unbleached control regions and normalized all results to correct for changes in control regions (e.g., Fig. $2 C$ ). For example, if a control region brightness decreased by $10 \%$ during the postbleach waiting period, it was assumed that full recovery of the bleached region also decreased by $10 \%$. Thus, in the example above, recovery from 100 to 150 would be $50 / 80$, or $62.5 \%$, rather than $50 \%$. Usually, control regions decreased in brightness slightly, probably as a result of slight focus

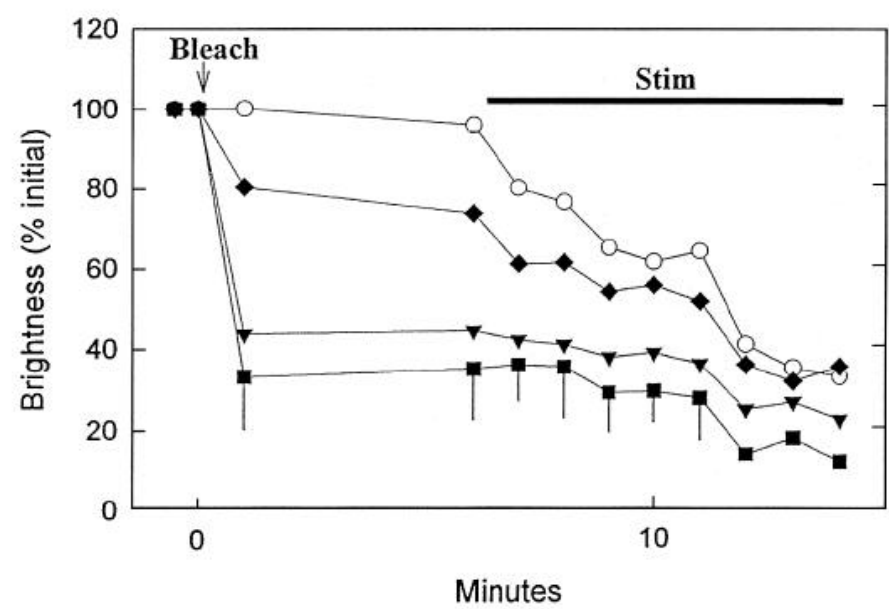

Figure 3. Averaged results from six experiments confirmed that no significant recovery from photobleaching occurred during nerve stimulation. Each filled symbol shows the average brightness of pixels lying a fixed distance from the center of the bleached region [distance $=0.5$ $\mu \mathrm{m}$ (squares), $1.0 \mu \mathrm{m}$ (triangles), and $1.8 \mu \mathrm{m}$ (diamonds)]. The open circles show the average brightness in several control regions, far away from the bleached spot. Pixels closest to the center of the bleached region (filled squares) were most completely bleached, whereas at slightly farther distances (triangles and diamonds), less bleaching occurred. A $5 \mathrm{~min}$ rest period followed the bleach, and then the nerve was stimulated continuously at 10 or $20 \mathrm{~Hz}$ while additional images were acquired. The center of the bleached region (filled squares) showed no significant recovery. Brightnesses were calculated as the average brightness of pixels lying along concentric circles centered on the bleached region (which was approximately circular). Vertical lines show -1 SEM for the center of the bleached regions; other SEs were of similar magnitude and are omitted for clarity.

changes and slight photobleaching during image acquisition. These effects would cause the amount of recovery from photobleaching to be somewhat overestimated and make it likely that the average measured recovery (18\% recovery $25-30$ min after bleaching) (Fig. 3 ) is not significantly different from zero recovery. Because the recovery was so meager, our attempts to quantify photobleach recovery rates in resting terminals were unsuccessful; estimates of diffusion constant were consistently less than $10^{-12} \mathrm{~cm}^{2} / \mathrm{sec}$.

\section{RESULTS}

\section{Recovery from photobleaching in resting terminals}

Figure $1 A$ shows the results of an experiment on a normal, resting nerve terminal. The control image (top) shows typical fluorescent spots in a nerve terminal (the arrowhead marks the region to be bleached). These spots, each of which consists of several hundred to a few thousand recycled synaptic vesicles stained with FM1-43, persist in resting terminals for many hours without significant changes in brightness, position, or shape (Betz et al., 1992a). The middle shows the same terminal immediately after a small patch was bleached with the laser. The image in the bottom was obtained $28 \mathrm{~min}$ after photobleaching; the bleached region still is evident (arrowhead). Similar results were obtained in experiments on 11 other muscles ( 25 terminals; 35 bleached spots and 5 bleached lines). Overall, 25-60 min after photobleaching there was only $\sim 18 \%$ recovery of brightness in the bleached regions, an amount that, owing to slight amounts of focus change and photobleaching during image acquisition (see Materials and Methods), may not be different from zero (i.e., completely immobilized vesicles).

\section{Effects of okadaic acid and other agents}

One simple explanation for the lack of recovery from photobleaching in resting nerve terminals is that the laser beam dam- 
aged the terminal and arrested vesicle movements that otherwise would have led to recovery from bleaching. We tested for this possibility in several ways. First, as illustrated in Figure $1 B$, we repeated the photobleach experiments on preparations pretreated with okadaic acid, an inhibitor of protein phosphatases (Haystead et al., 1989). In separate work (Betz and Henkel, 1994), we showed that okadaic acid disrupts synaptic vesicle clusters and causes vesicles to spread throughout the terminal. The dye movements do not appear to be diffusion-driven. Rather, it appears that okadaic acid unmasks powerful and widespread active translocators that move vesicles bidirectionally in the terminals. If the laser beam caused nonspecific damage to the cytoplasm and immobilized organelles, then one might expect that okadaic acid-treated preparations would not recover from photobleaching. This was not observed. As shown in Figure $1 B$, significant and prompt recovery from photobleaching occurred in terminals pretreated with okadaic acid. The images in Figure $1 B$ are like those in Figure $1 A$, except that the time interval between bleaching and the last image in the series was only $5 \mathrm{~min}$. It is clear that significant recovery from photobleaching occurred during this $5 \mathrm{~min}$ period. Note, for example, that in the bottom of Figure $1 B$, the bleached region (arrowhead) clearly is brighter than it was immediately after bleaching (middle). This prompt recovery from photobleaching was typical in preparations treated with okadaic acid but was never seen in control preparations. In other experiments, we found that okadaic acid, applied after photobleaching, also could mobilize vesicles and lead to recovery in photobleached regions (data not shown).

We tested the effects of several agents on the recovery from photobleaching induced by okadaic acid. Results are summarized in Figure $1 C$, which shows mean $(+1 \mathrm{SEM})$ percentage of photobleach recovery under several different conditions. The two left columns show results from control and okadaic acid-treated preparations, as illustrated in Figures $1 A, B$. The next three columns show, respectively, that cytochalasin $\mathrm{D}(\mathrm{CD})$, which disrupts actin polymerization and depolymerization (Cooper, 1987), did not block the okadaic acid effect, but that calmidozolium (Calmid), a calmodulin inhibitor, and staurosporine (Ssp), a protein kinase inhibitor, did block recovery from photobleaching induced by okadaic acid. By themselves, CD, Calmid, nor Ssp promoted recovery from photobleaching (data not shown).

\section{Effect of nerve stimulation on recovery from photobleaching}

As illustrated in Figure 2, repetitive nerve stimulation did not promote recovery from photobleaching. First, a control image was taken (Fig. 2A). Then the center of one spot was bleached almost completely (Fig. $2 B$, arrowhead). Additional images were acquired during a $5 \mathrm{~min}$ rest period and during a subsequent period of repetitive nerve stimulation $(10 \mathrm{~Hz}$ for $5 \mathrm{~min}$ ); the last image in this series is shown in Figure $2 C$. Note that after nerve stimulation, all of the fluorescent spots were dimmer, reflecting the activity-dependent destaining of the terminal. The bleached region also is less evident, which might suggest that recovery from photobleaching had occurred during the nerve stimulation. However, as shown in Figure $2 D$ (which shows the brightness of pixels under a line drawn along the length of the terminal, through the center of fluorescent spots, at different times; the bleached region is shaded), the bleached region did not grow brighter, the surround simply grew dimmer during repetitive nerve stimulation. Thus, there was no net movement of dye into the bleached region during or after nerve stimulation. In addition, the bleached region did not grow dimmer during nerve stimulation, probably because the bleach was nearly complete. We examined the ability of lightly bleached terminals to destain in other experiments (see Fig. 7).

We also measured the average brightness of pixels lying a fixed distance from the center of the bleached spot. Averaged results from six experiments are shown in Figure 3. The brightness near the center of the bleached region (filled squares), closer to the periphery of the bleached region (triangles and diamonds), and in remote control areas (open circles) is plotted. It is clear that on average, the bleached regions showed virtually no recovery after nerve stimulation, and in no individual case did we observe significant brightening in a bleached region.

\section{Effects of botulinum toxin}

These results suggest that, even during repetitive nerve stimulation, synaptic vesicles are not free to move laterally within a vesicle cluster. However, another possibility is that mobilized vesicles did move into the bleached region, but then underwent exocytosis and lost their dye, so that the recovery from photobleaching was incomplete and transient and was undetected by us. To test this possibility, we repeated the nerve stimulation experiments on preparations that had been poisoned with botulinum toxin, a potent inhibitor of exocytosis that acts by cleaving peptides involved in synaptic vesicle docking at the presynaptic membrane (for review, see Simpson, 1989; Montecucco and Schiavo, 1994). Consistent with this interpretation, we found that botulinum toxin blocked activity-dependent destaining of nerve terminals that had been preloaded with FM1-43 (serotypes A, C, and D gave indistinguishable results). Results from a typical experiment are shown in Figure 4. We also found, as expected, that pretreatment with botulinum toxin blocked subsequent staining of nerve terminals with FM1-43 (data not shown). The block of destaining could be overcome by treatment with black widow spider venom (Fig. 5A). Botulinum toxin treatment did not, however, block the vesicle-mobilizing actions of okadaic acid (Betz and Henkel, 1994). As illustrated in Figure $5 B$, vesicle clusters were disrupted by okadaic acid $(2 \mu \mathrm{M})$ in preparations pretreated with botulinum toxin to an extent that was indistinguishable from controls. Okadaic acid treatment, however, did not overcome the botulinum toxin-induced block of destaining by nerve stimulation (data not shown). In summary, these results are entirely consistent with evidence that botulinum toxin acts by blocking vesicle docking.

Next, we repeated the photobleach experiments on botulinumpoisoned preparations (Fig. 6). After a control image was acquired (Fig. 6A), two spots were bleached (Fig. 6B, arrowheads). Then the nerve was stimulated ( $30 \mathrm{~Hz}$ for $5 \mathrm{~min}$ ), and the image in Figure $6 C$ was acquired. It is clear that destaining was blocked (compare Fig. 4) and that little or no recovery from photobleaching occurred. Figure $6 D$ shows the profile of brightness for the spot on the right obtained from the images in $A-C$. Figure $6 E$ shows averaged results from six experiments. The centers of the bleached spots were aligned on the $x$-axis $(x-0)$. The top line (solid) shows the mean brightness +1 SEM for all six experiments before bleaching. The dotted line shows the same for spots immediately after bleaching and the dashed line after nerve stimulation. For clarity, error bars, which were about the same length as controls, are not shown. The two postbleach lines are nearly identical, showing that virtually no vesicle mixing occurred during stimulation of botulinum-poisoned terminals.

\section{Destaining of partially bleached regions}

Once an area was bleached, we, of course, could not monitor directly the behavior of the bleached vesicles. However, we per- 

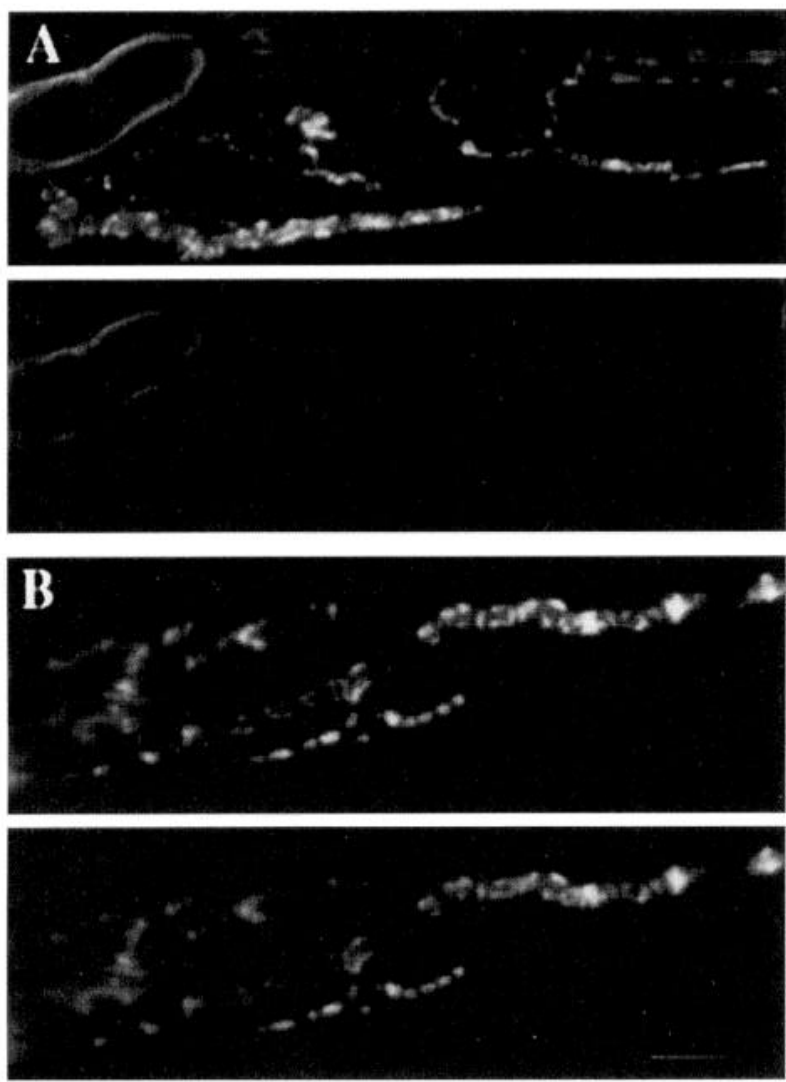

Figure 4. Botulinum toxin blocked destaining of frog motor nerve terminals preloaded with FM1-43. Preparations were stained with FM1-43, and then one $(B)$ was exposed to botulinum toxin (type D) $(10 \mathrm{nM})$ for $4 \mathrm{hr}$. Then the prestimulation images (top) were acquired. Next, the nerves to each preparation were stimulated $(10 \mathrm{~Hz}$ for $10 \mathrm{~min})$, and images again were taken (bottom). The control terminal $(A)$ destained during the nerve stimulation, whereas the botulinum toxin-poisoned terminal $(B)$ did not. In the control panels, a myelinated axon is visible in the upper left corner. Scale bar, $5 \mu \mathrm{m}$.

formed several additional tests to examine the overall functional integrity of bleached regions. First, we used smaller amounts of bleaching (shorter bleaching periods) than in the experiments shown in Figures 4-6 to test for the ability of a partially bleached region to destain during nerve stimulation. An example is shown in Figure 7. The graph shows the profile of brightness along the length of the terminal (compare Fig. 6D) before bleaching (solid line), immediately after bleaching (dotted line), and after repetitive nerve stimulation (10 Hz for $5 \mathrm{~min}$ ) (dashed line). Bleaching reduced the brightness by $\sim 40 \%$ (shaded region). Subsequent nerve stimulation produced complete destaining of the partially bleached region. In most experiments, the rate of destaining in the bleached region was slower than normal, as reported previously for overillumination using a mercury lamp for illumination (Betz et al., 1992).

\section{Restaining and destaining in bleached regions}

We performed two additional tests for laser-induced photodamage. Typical results are shown in Figure 8 . First, restaining of bleached regions was successful (Fig. $8 A, 3$ ). Second, destaining of those restained areas also was successful (4). These tests showed that exocytotic, endocytotic, and vesicle recycling machinery was intact qualitatively after the laser illumination.

\section{A Control}
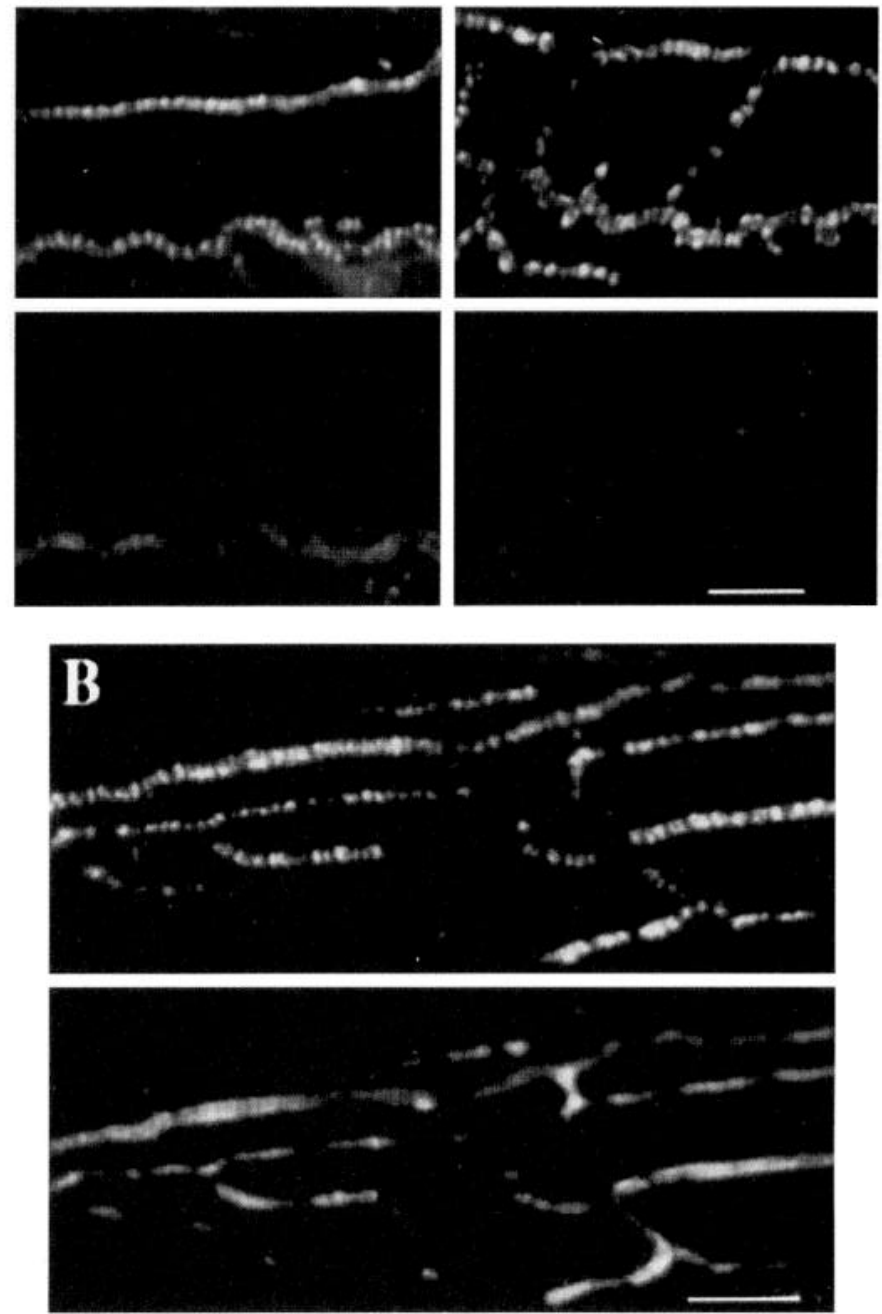

Figure 5. Botulinum toxin did not block the actions of black widow spider venom or okadaic acid. $A$, These preparations were stained with FM1-43, and then one (right) was poisoned with botulinum toxin (type C) $(10 \mathrm{~nm}$ for $3 \mathrm{hr})$. The control images (top) show fluorescent spots characteristic of normal staining. Then black widow spider venom $(0.3$ glands $/ \mathrm{ml}$ ) was applied for $40 \mathrm{~min}$, and the images (bottom) were acquired. It is clear that black widow spider venom caused destaining in control and botulinum-poisoned terminals alike. Scale bar, $10 \mu \mathrm{m} . B$, The control image (top) shows a terminal in a preparation that was stained with FM1-43 and then poisoned with botulinum toxin (type C) (10 $\mathrm{nm}$ for $3 \mathrm{hr}$ ). Then $5 \mu \mathrm{M}$ okadaic acid was applied for $120 \mathrm{~min}$, and the terminal was reimaged (bottom). The blurring of fluorescent spots is clearly apparent. This is an extreme example of the okadaic acid effect, probably resulting from the prolonged exposure to okadaic acid. In general, blurring of dye spots could be seen after exposure to okadaic acid for $30-40 \mathrm{~min}$. Scale bars, $10 \mu \mathrm{m}$.

\section{DISCUSSION}

In top views of both resting and stimulated nerve terminals, little or no recovery from photobleaching occurred, suggesting that synaptic vesicles are virtually immobile in resting terminals and that their lateral movements are restricted sharply as they make their way to the presynaptic membrane during repetitive nerve stimulation. These conclusions rest on the assumption that laser photodamage was negligible. In frog motor nerve terminals stained with FM1-43, overillumination can cause immobilization 


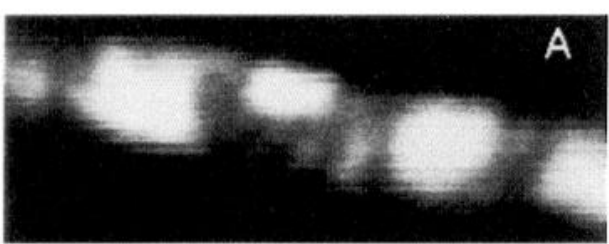

A
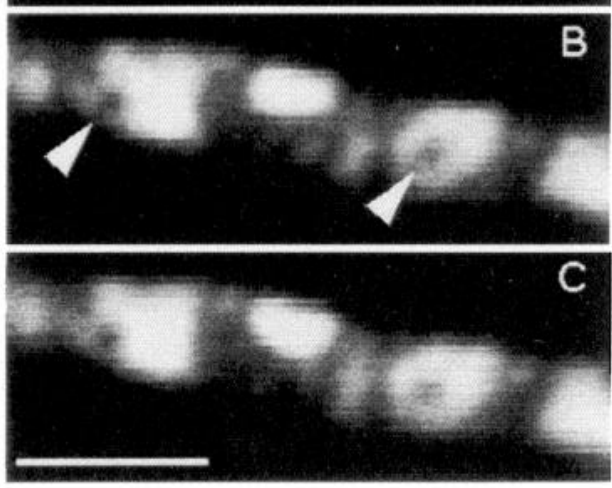
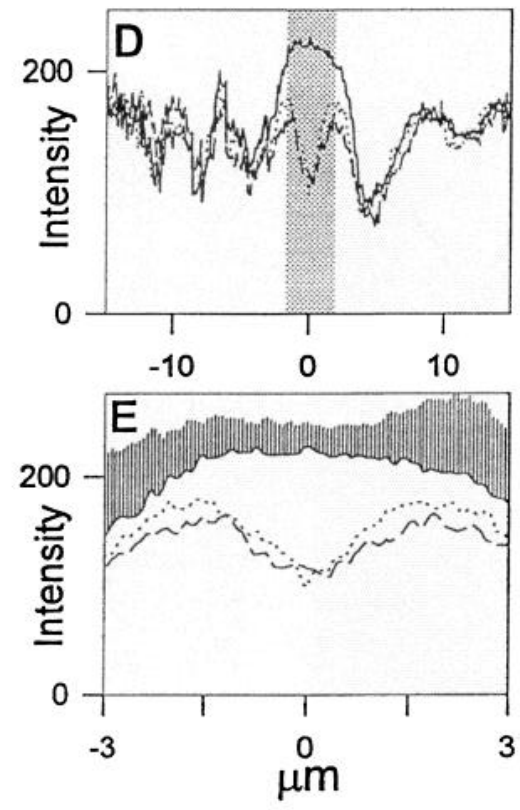

Figure 6. Fluorescence photobleach recovery did not occur in botulinum toxin-poisoned preparations. $A$, A nerve terminal stained with FM1-43 and poisoned with botulinum toxin before photobleaching. $B$, The same terminal immediately after photobleaching (arrowheads mark bleached regions). $C$, After repetitive nerve stimulation $(30 \mathrm{~Hz}$ for $5 \mathrm{~min})$. Little or no change occurred as a result of the nerve stimulation. Scale bar, $5 \mu \mathrm{m}$. $D$, Profile of brightness along a line passing through the bleached region for the images in $A-C$. $E$, Averaged results from six different experiments with centers of bleached regions aligned at $x=0$. Solid line shows mean +1 SEM before bleaching. Results obtained immediately after bleaching and after repetitive nerve stimulation are shown with dotted and dashed lines, respectively. Error bars, which were about the same length as before bleaching, are omitted from these two lines for clarity. of synaptic vesicles, blocking activity-dependent destaining of the terminals (Betz et al., 1992a). This made it crucial to determine whether the observed lack of recovery from photobleaching was attributable simply to photodamage. We performed four different tests to study this problem, and the results, taken together, suggest that photodamage is not the correct explanation for the lack of recovery from photobleaching.

The protein phosphatase inhibitor okadaic acid provided one test for photodamage. After FM1-43-stained preparations had been exposed to okadaic acid, recovery from photobleaching was brisk, beginning immediately and sometimes going to completion within several minutes. In previous work (Betz and Henkel, 1994), we showed that okadaic acid unmasks a vesicle translocation mechanism in nerve terminals. The present results show clearly that laser photobleaching did not interfere with these movements.

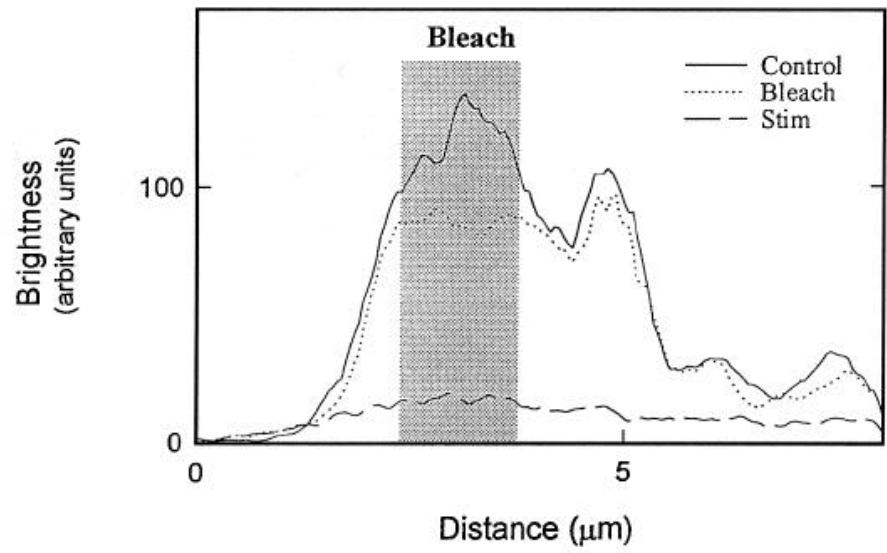

Figure 7. Partially bleached regions can be destained completely by nerve stimulation. The profile of brightness through a single fluorescent spot is plotted as in Figure $6 D$. The solid line shows the profile before photobleaching. The extent of photobleaching was reduced by shortening the duration of exposure to the laser. In this case, the fluorescent spot was bleached (shaded region) by only $30-40 \%$ (dotted line). Then the nerve was stimulated ( $10 \mathrm{~Hz}$ for $5 \mathrm{~min})$. The spot destained completely (dashed line). This shows that vesicles located in the center of a bleached region still can undergo destaining.
We do not know whether the same mechanism moves synaptic vesicles to the presynaptic membrane during ordinary nerve activity; it is conceivable that a different mechanism, more sensitive to laser illumination, might exist. Nevertheless, the persistence after photobleaching of the okadaic acid effect shows that the laser did not produce generalized nonspecific damage to the cytoplasm.

Three additional tests demonstrated that photobleaching did not destroy the cytoplasmic machinery necessary for carrying out the complex tasks of exocytosis and vesicle recycling. First, after a "light" amount of bleaching, the remaining dye still could be released in an activity-dependent fashion, although the rate of destaining was somewhat reduced compared with controls. This showed that exocytosis was at least qualitatively intact after photobleaching. Second, bleached regions could be restained with FM1-43. Third, bleached, reloaded terminals could be destained completely by nerve stimulation. Collectively, these experiments show that the machinery necessary for vesicle mobilization, exocytosis, endocytosis, and recycling was qualitatively intact in bleached regions. We cannot, however, rule out the possibility that the laser selectively destroyed a mechanism specifically designed for the lateral movement of vesicles within vesicle clusters. It seems more reasonable to conclude that, although photobleaching may have produced a partial reduction in the rate of vesicle movements, it did not block them altogether. We, thus, sought other explanations for the lack of recovery from photobleaching.

One possibility is that during repetitive nerve stimulation, dye-filled vesicles did in fact move into bleached regions, but then quickly underwent exocytosis, losing their dye, thereby producing only a transient, undetected recovery from photobleaching. To test this, we monitored bleached regions after stimulation had ended, when exocytosis, but probably not mobilization, had been arrested, and we still saw no significant photobleach recovery. In addition, we repeated photobleach experiments on preparations poisoned with botulinum toxin (which blocks exocytosis and, therefore, dimming of fluorescence, but not vesicle mobilization), with the same result. Thus, 

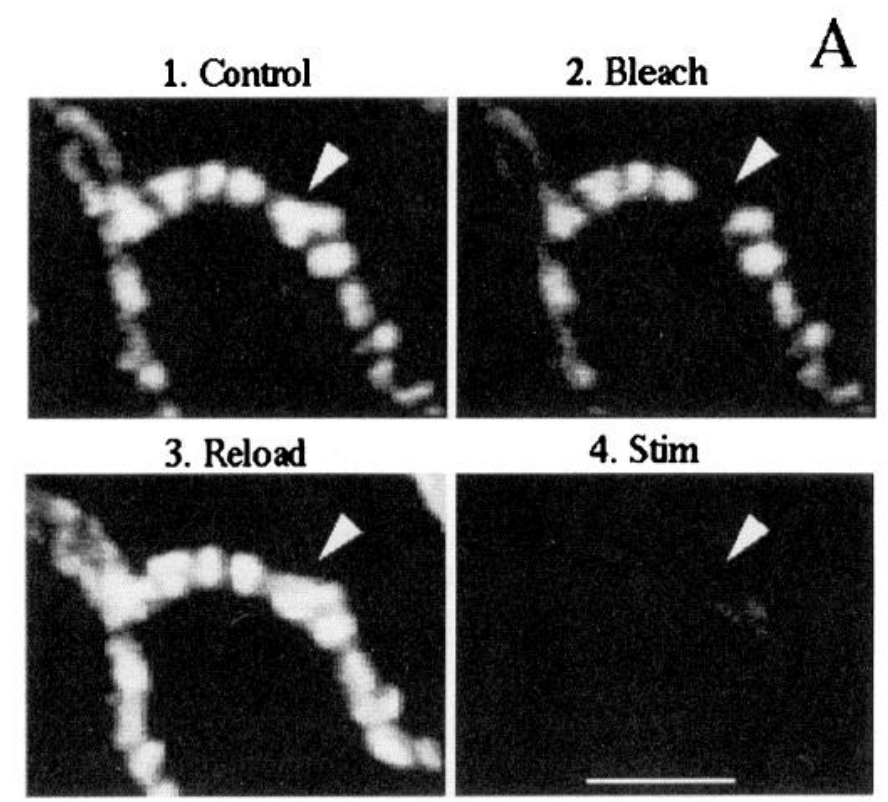

\section{Stim}
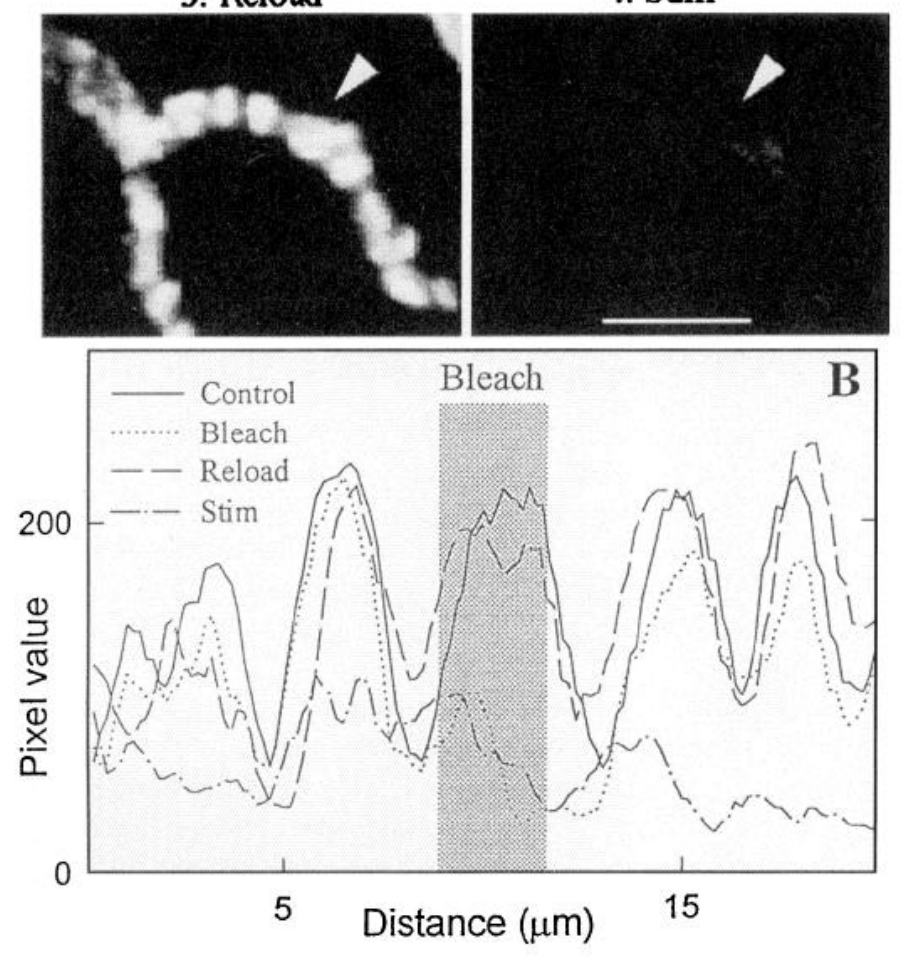

Figure 8. Photobleached regions exhibit normal functional properties, including restaining and destaining with FM1-43. $A$, Images of a normal terminal (1) that was bleached (2, arrowhead), restained with FM1-43 (3), and then destained by nerve stimulation $(30 \mathrm{~Hz}$ for $5 \mathrm{~min})(4)$. The restaining and destaining of the previously bleached region were indistinguishable from control regions. Scale bar, $10 \mu \mathrm{m}$. $B$, Brightness profiles of the images in Figure $8 A$.

it seems that the results reflect a true failure of vesicles to move laterally within vesicle clusters.

This conclusion seems, at first consideration, to contradict previous results. For example, recycled vesicles appear to mix randomly with preexisting vesicles, as measured by horseradish peroxidase (for review, see Heuser, 1989) or FM1-43 uptake and distribution (Betz and Bewick, 1992). Such mixing occurs at an earlier phase of the vesicle cycle, namely, the period between endocytosis and reappearance of vesicles in the cluster. It is possible that the mechanism responsible for generating nascent vesicles can somehow "inject" them randomly into a cluster of otherwise immobilized vesicles. Also, observations of side views of nerve terminals before and after partial destaining suggested that some fluorescent spots dimmed uniformly during nerve stimulation, as if vesicles were mixing freely within a cluster (Betz et al., 1992b). However, directed or constrained vesicle movements were not ruled out by those observations; bidirectional movements of vesicles confined to pathways oriented perpendicular to the presynaptic membrane could produce results consistent with both studies. In the present work, we attempted to resolve this question by bleaching portions of dye spots viewed from the side, rather than from the top, but we were not successful, owing to their small size when viewed from the side.

How might synaptic vesicles be held even as they move to the presynaptic membrane? Perhaps the simplest explanation is that each vesicle is tethered to its neighbors and is simply pulled forward as its cohorts undergo exocytosis and collapse into the presynaptic membrane. Alternatively, the theory of action of synapsin I (for review, see DeCamilli et al., 1990), a protein that binds synaptic vesicles in resting terminals (Landis et al., 1988; Hirokawa et al., 1989), but dissociates from vesicles after being phosphorylated appropriately (Valtorta et al., 1992; Greengard et al., 1993), would require that some constraining force persist or be added after a vesicle is freed from synapsin I. The identity of this constraint is unknown. Some studies suggest a role for actin in vesicle binding in nerve terminals (Hirokawa et al., 1989; Benfenati et al., 1992; Valtorta et al., 1992), whereas others are not wholly consistent with such a role (Landis et al., 1988; Nakata and Hirokawa, 1992). In chromaffin cells, actin is postulated to be a barrier to mobilization and docking of granules (Aunis and Bader, 1988; Sontag et al., 1988; Vitale et al., 1995). In the present work, CD did not affect recovery from photobleaching, suggesting at least that changes in actin polymerization (Cooper, 1987) are not involved in the movement of synaptic vesicles to the presynaptic membrane in frog motor nerve terminals.

\section{REFERENCES}

Aunis D, Bader MF (1988) The cytoskeleton as a barrier to exocytosis in secretory cells. J Exp Biol 139:253-266.

Axelrod D, Koppel DE, Schlessinger J, Elson E, Webb WW (1976) Mobility measurement by analysis of fluorescence photobleaching recovery kinetics. Biophys J 16:1055-1069.

Benfenati F, Valtorta F, Chieregatti E, Greengard P (1992) Interaction of free and synaptic vesicle-bound synapsin I with F-actin. Neuron 8:377-386.

Betz WJ, Bewick GS (1992) Optical analysis of synaptic vesicle recycling at the frog neuromuscular junction. Science 255:200-203.

Betz WJ, Bewick GS (1993) Optical monitoring of transmitter release and synaptic vesicle recycling at the frog neuromuscular junction. J Physiol (Lond) 460:287-309.

Betz WJ, Henkel AW (1994) Okadaic acid disrupts clusters of synaptic vesicles in frog motor nerve terminals J Cell Biol 124:843-854.

Betz WJ, Mao F, Bewick GS (1992a) Activity-dependent fluorescent staining and destaining of living vertebrate motor nerve terminals. J Neurosci 12:363-375.

Betz WJ, Bewick GS, Ridge RMAP (1992b) Intracellular movements of fluorescently labeled synaptic vesicles in frog motor nerve terminals during nerve stimulation. Neuron 9:805-813.

Betz WJ, Ridge RMAP, Bewick GS (1993) Comparison of FM1-43 staining patterns and electrophysiological measures of transmitter release at the frog neuromuscular junction. J Physiol (Paris) 87:193-201.

Bewick GS, Betz WJ (1994) Illumination partly reverses the postsynaptic blockade of the frog neuromuscular junction by the styryl pyridinium dye RH414. Proc R Soc Lond [Biol] 258:201-207.

Cooper JA (1987) Effects of cytochalasin and phalloidin on actin. J Cell Biol 105:1473-1478.

DeCamilli P, Benfenati F, Valtorta F, Greengard P (1990) The synapsins. Annu Rev Cell Biol 6:433-460.

Greengard P, Valtorta F, Czernik AJ, Benfenati F (1993) Synaptic vesicle phosphoproteins and regulation of synaptic function. Science 259:780-785.

Haystead TAJ, Sim ATR, Carling D, Honnor RC, Tsukitani R, Cohen P, Hardie DG (1989) Effects of the tumour promoter okadaic acid on intracellular protein phosphorylation and metabolism. Nature 337:78-81. 
Henkel AW, Betz WJ (1995) Monitoring of black widow spider venom (BWSV) induced exo- and endocytosis in living frog motor nerve terminals with FM1-43. Neuropharmacology 34:1397-1406.

Hcnkel AW, Lübkc J, Bctz WJ (1996) FM1-43 ultrastructural localization in and release from frog motor nerve terminals. Proc Natl Acad Sci USA 93:1918-1923.

Heuser J (1989) Review of electron microscopic evidence favouring vesicle exocytosis as the structural basis for quantal release during synaptic transmission. O J Exp Physiol 74:1051-1069.

Hirokawa N, Sobuc K, Kanda K, Harada A, Yorifuji H (1989) The cytoskeletal architecture of the presynaptic terminal and molecular structurc of synapsin. Int J Ccll Biol 108:111-126.

Jacobson K, Zhang F, Tsay TT (1991) Fluorescence recovery after photobleaching techniques to measure translational mobility in microscopic samples. Scanning Microsci 5:357-362.

Landis DMD, Hall AK, Weinstein LA, Reese TS (1988) The organization of cytoplasm at the presynaptic active zone of a central nervous system synapse. Neuron 1:201-209.

Montecucco C, Schiavo G (1994) Mechanism of action of tetanus and botulinum neurotoxins. Mol Microbiol 13:1-8.

Nakata T, Hirokawa N (1992) Organization of cortical cytoskeleton of cultured chromaffin cells and involvement in secretion as revealed by quickfreeze, deep-etching, and double-label immunoelectron microscopy. J'Neurosci 12:2186-2197.
Ribchester RR, Betz W.I (1994) Optical measurements of activitydependent membrane recycling in motor nerve terminals of mammalian skeletal muscle. Proc R Soc Lond [Biol] 255:61-66.

Simpson LL (1989) Botulinum neurotoxin and tetanus toxin. San Diego: Academic.

Simpson LL, Dasgupta BR (1983) Botulinum neurotoxin type E: studies un the mechanism of action and on structure-activity relationships. J Pharmacol Exp Ther 224:135-140.

Simpson LL, Schmidt JJ, Middlebrook JL (1988) Isolation and characterization of the botuinum neurotoxins. Methods Enzymol 165:76-85.

Sontag JM, Aunis D, Bader MF (1988) Peripheral actin filaments control calcium-mediated catecholamine release from streptolysin-Opermeabilized chromaffin cells. Eur J Cell Biol 46:316-326.

Terakawa S, Manivannan S, Kumakura K (1993) Evidence against the swelling hypothesis for initiation of exocytosis in terminals of chromaffin cell processes. J Physiol (Paris) 87:209-213.

Valtorta F, Greengard P, Fesce R, Chieregatti E, Benfenati F (1992) Effects of the neuronal phosphoprotein synapsin I on actin polymerization. J Biol Chem 267:11281-11299.

Vitale ML, Seward EP, Trifaro J-M (1995) Chromaffin cell cortical actin network dynamics control the size of the release-ready vesicle pool and the initial rate of exocytosis. Neuron 14:353-363.

Wade MH, Trosko JE, Schindler MA (1986) A fluorescence photobleaching assay of gap junction-mediated communication between human cells. Science 232:525-528. 